\title{
Altered prostanoid metabolism contributes to impaired angiogenesis in persistent pulmonary hypertension in a fetal lamb model
}

\author{
Chaitali N. Mahajan' ${ }^{1}$ Adeleye J. Afolayan², Annie Eis², Ru-Jeng Teng² and Girija G. Konduri²
}

BACKGROUND: Persistent pulmonary hypertension of the newborn (PPHN) is associated with decreased lung angiogenesis and impaired pulmonary vasodilatation at birth. Prostanoids are important modulators of vascular tone and angiogenesis. We hypothesized that altered levels of prostacyclin $\left(\mathrm{PGI}_{2}\right)$, a potent vasodilator, and thromboxane $\mathrm{A}_{2}\left(\mathrm{TX} \mathrm{A}_{2}\right)$, a vasoconstrictor, contribute to impaired angiogenesis of pulmonary artery endothelial cells (PAEC) in PPHN.

METHODS: PAEC were isolated from fetal lambs with PPHN induced by prenatal ductus arteriosus constriction or from sham operated controls. Expression and activity of $\mathrm{PGI}_{2}$ synthase (PGIS) and TXA synthase (TXAS), expression of cyclooxygenases 1 and 2 (COX-1 and COX-2), and the role of PGIS/TXAS alterations in angiogenesis were investigated in PAEC from PPHN and control lambs.

RESULTS: PGIS protein and activity were decreased and PGIS protein tyrosine nitration was increased in PPHN PAEC. In contrast, TXAS protein and its stimulated activity were increased in PPHN PAEC. COX-1 and COX-2 proteins were decreased in PPHN PAEC. Addition of $\mathrm{PGl}_{2}$ improved in vitro tube formation by PPHN PAEC, whereas indomethacin decreased tube formation by control PAEC. PGIS knockdown decreased the in vitro angiogenesis in control PAEC, whereas TXAS knockdown increased the in vitro angiogenesis in PPHN PAEC.

CONCLUSION: Reciprocal alterations in $\mathrm{PGI}_{2}$ and $T X A_{2}$ may contribute to impaired angiogenesis in PPHN.

$\mathrm{P}$ ersistent pulmonary hypertension of the newborn (PPHN) represents a failure of the normal postnatal adaptation that occurs at birth in pulmonary circulation. It is characterized by decreased blood vessel density in the lungs (1) and impaired pulmonary vasodilatation at birth, both of which lead to postnatal persistence of high pulmonary vascular resistance. The increased pulmonary vascular resistance can result from a decrease in vasodilator signals, or increase in vasoconstrictor signals, by pulmonary artery endothelial cells (PAEC) in PPHN. Nitric oxide (NO) and prostacyclin $\left(\mathrm{PGI}_{2}\right)$ are two key mediators involved in pulmonary vasodilatation at birth (2-4). Although alterations in NO-cyclic guanosine monophosphate system have been extensively studied in PPHN, the role of altered prostanoid signaling in PPHN remains unclear. Inhaled NO therapy has improved the outcomes in PPHN; however, some neonates do not respond to this therapy (5). Impaired vascular growth in the lung may contribute to this failure of response to $\mathrm{NO}(6)$.

$\mathrm{PGI}_{2}$ is a prostanoid synthesized from arachidonic acid through cyclooxygenase (COX)-PGI synthase (PGIS) pathway. $\mathrm{PGH}_{2}$, the catalytic end product of COX activity and a vasoconstrictor itself, is further metabolized by PGIS to $\mathrm{PGI}_{2}$. $\mathrm{PGI}_{2}$ is synthesized primarily in vascular cells, especially in the vascular endothelium (7). $\mathrm{PGI}_{2}$ causes vasodilatation by activating adenylate cyclase in the vascular smooth muscle cells via a G-protein-coupled receptor, which increases cyclic adenosine monophosphate synthesis. A surge in $\mathrm{PGI}_{2}$ in pulmonary circulation during perinatal transition contributes to pulmonary vasodilatation at birth (3). Regulation of PGIS, which directs the synthesis of $\mathrm{PGI}_{2}$, during fetal life and its alterations in PPHN remain unclear. $\mathrm{PGI}_{2}$ also modulates blood vessel formation (8), and decreases in $\mathrm{PGI}_{2}$ levels may lead to impaired angiogenesis in PPHN. However, the role of $\mathrm{PGI}_{2}$ as a mediator of angiogenesis during perinatal transition remains unexplored. Thromboxane $\mathrm{A}_{2}\left(\mathrm{TXA}_{2}\right)$, another arachidonic acid metabolite generated from $\mathrm{PGH}_{2}$ by thromboxane synthase (TXAS), is a potent pulmonary vasoconstrictor (9), particularly during hypoxia. TXA is believed to promote angiogenesis during inflammation, but its effect on angiogenesis in developing lungs is also unknown. An imbalance between $\mathrm{PGI}_{2}$ and TXA $\mathrm{TH}_{2}$ may be involved in the pathogenesis of PPHN. Previous studies have shown that impaired $\mathrm{PGI}_{2}$ signaling leads to impaired vasodilation in the ductal constriction model of PPHN (10). However, the role of altered prostaglandin signaling in impaired angiogenesis in PPHN has not been previously investigated. Oxidative stress impairs vasodilatation and angiogenesis $(11,12)$ in PPHN and may modulate the release of prostanoids in PPHN $(13,14)$. 
We hypothesized that PPHN is associated with an altered balance of $\mathrm{PGI}_{2}$ and $\mathrm{TXA}_{2}$, which in turn leads to impaired angiogenesis. We investigated our hypothesis in the fetal lamb model of PPHN induced by intrauterine ductal constriction.

\section{RESULTS}

\section{$\mathrm{PGI}_{2}$ and PGIS Alterations in PPHN}

Basal levels of 6-Keto-PGF1 $\alpha$, a stable metabolite of PGI, were decreased by fourfold in PAEC from PPHN lambs when compared with control cells (Figure 1a; $n=12 ; P<0.05$ ). Adenosine triphosphate (ATP)-stimulated 6-Keto-PGF1 $\alpha$ ( $\mathrm{PGI}_{2}$ metabolite) levels were also significantly lower in PPHN than that in control cells. PGIS protein levels were decreased by 2.5 -fold in PPHN compared with control PAEC (Figure $1 \mathbf{b} ; n=14$; $P<0.05)$. PGIS mRNA levels were not different between control and PPHN cells (Figure 1c; $n=7 ; P=0.12$ ). Tyrosine nitration of PGIS was increased in PPHN cells by twofold compared with control PAEC (Figure 1d; $n=6 ; P<0.05$ ).
TXA $_{2}$ Levels and TXAS Expression in PPHN

ATP-stimulated levels of $\mathrm{TXB}_{2}$, a stable metabolite of TXA, were increased by twofold in PAEC from PPHN lambs compared with control PAEC (Figure 2a; $n=11 ; P<0.05$ ), although basal levels of $\mathrm{TXB}_{2}$ were not different (data not shown). TXAS protein levels were increased in PPHN cells compared with controls (Figure $2 \mathbf{b} ; n=14 ; P<0.05$ ). TXAS mRNA abundance was significantly decreased in PAEC from PPHN lambs compared with control lambs (Figure 2c; $n=9 ; P<0.05$ ).

\section{COX-1 and COX-2 Expression in PPHN}

COX-1 protein levels were decreased in PPHN compared with control PAEC (Figure 3a; $n=11 ; P<0.05$ ). COX-1 mRNA levels were not different between PPHN and control PAEC (Figure 3c; $n=8 ; P=0.26$ ). COX-2 protein levels were decreased in PPHN compared with control PAEC (Figure 3b; $n=11 ; P<0.05)$. COX-2 mRNA levels were also not different between PPHN and control PAEC (Figure 3d; $n=8 ; P=0.67$ ). a

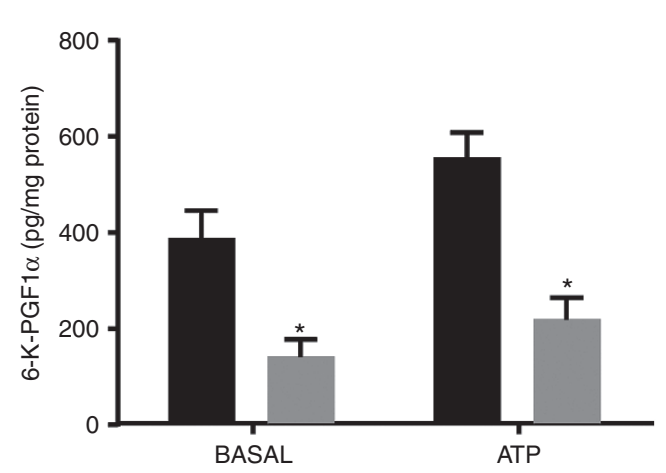

c

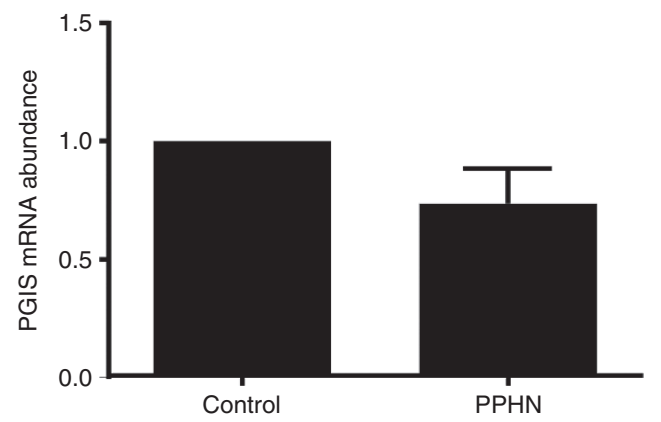

b
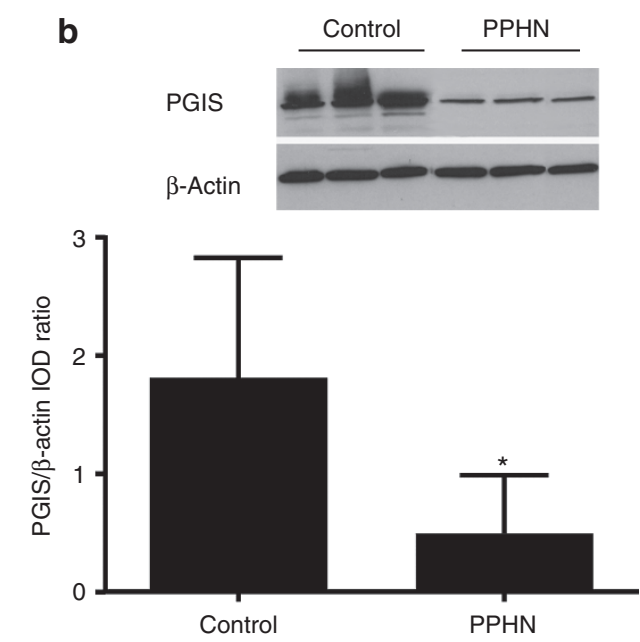

d
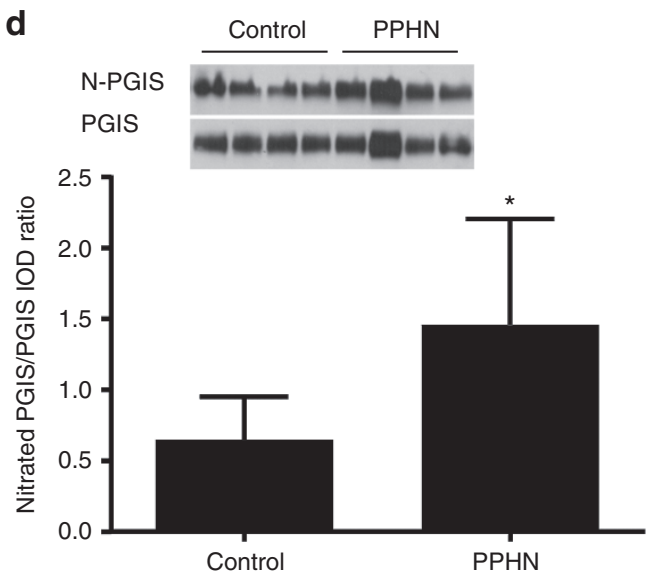

Figure 1. Alterations in prostacyclin synthase (PGIS) protein levels and activity in pulmonary artery endothelial cells (PAEC) in persistent pulmonary hypertension of the newborn (PPHN). (a) Levels of 6-Keto-PGF1 $\alpha$, a stable metabolite of PGI2, were decreased in PPHN (gray bar) at basal level and after ATP stimulation ( $n=12 ; P<0.05$ from control PAEC) compared with control cells (filled bar). (b) PGIS protein levels were decreased in PPHN ( $n=14$; $P<0.05)$. Representative western blots were shown with $B$-actin used as loading control. (c) PGIS mRNA levels were not different between PPHN and control PAEC $(n=7 ; P=0.12)$. (d) PGIS nitration was higher in PPHN than that in control PAEC. $(n=6 ; P<0.05)$. ${ }^{*} P<0.05$ compared with control PAEC. 


\section{In Vitro Angiogenesis in PPHN}

Total tube length was decreased in PPHN compared with control PAEC (Figure 4a,b), as we reported previously (12). When indomethacin was added to control PAEC, the tube length and branch point number were decreased (Figure 4b,c). Exogenous $\mathrm{PGI}_{2}$ improved tube formation in indomethacin-treated cells (Figure $4 \mathrm{a}-\mathrm{c}$ ). $\mathrm{PGI}_{2}$ also restored tube length and branch point number in PAEC from PPHN lambs to the same level as control PAEC (Figure 4b,c; $n=4$; $P<0.05)$.
Effect of PGIS Knockdown on In Vitro Angiogenesis

Tube length decreased by fourfold and branch point number decreased by eightfold when PGIS expression was knocked down by siRNA in control PAEC when compared with nonsilencing RNA (NS-siRNA) treated PAEC (Figure 5a; $n=6$; $P<0.05)$. The gap in PAEC with PGIS knockdown, $6 \mathrm{~h}$ after a scratch was $562.5 \pm 17.3 \mu \mathrm{m}$ compared with $347.5 \pm 9.5 \mu \mathrm{m}$ for NS-siRNA cells, indicating slower recovery from injury (Figure $5 \mathbf{b} ; n=10 ; P<0.05$ ). On cell invasion assay, $74.8 \pm 6.4$ of PGIS knockdown cells invaded the membrane as compared a

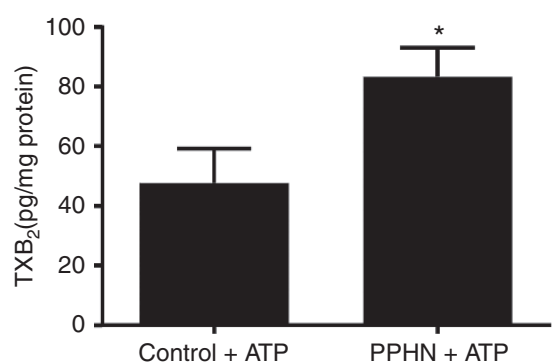

b
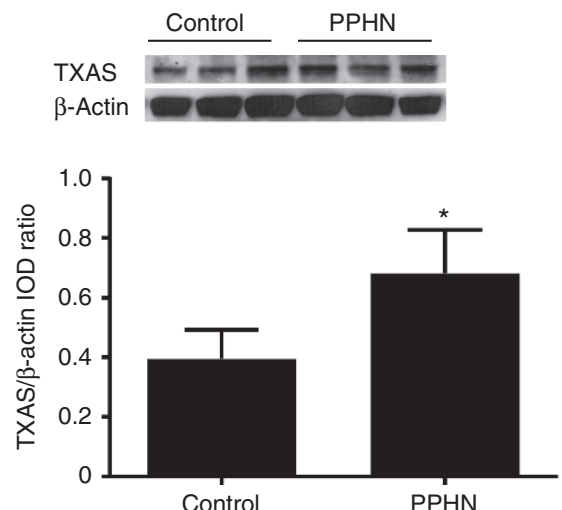

C

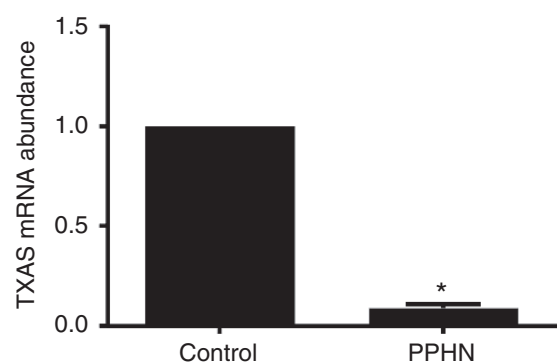

Figure 2. Alterations in protein level and activity of thromboxane $A 2$ synthase (TXAS) in PPHN PAEC. (a)Thromboxane $B_{2}\left(T_{X B_{2}} \text {, stable metabolite of TXA }\right)_{2}$ levels were increased with ATP stimulation in PPHN cells $(n=11 ; P<0.05$ from control PAEC). (b) TXAS protein levels were increased in PPHN cells ( $n=14$; $P<0.05)$. In contrast, (c) TXAS mRNA levels were decreased in PPHN cells $(n=11 ; P<0.05) .{ }^{*} P<0.05$ compared with control. PPHN, persistent pulmonary hypertension of the newborn.
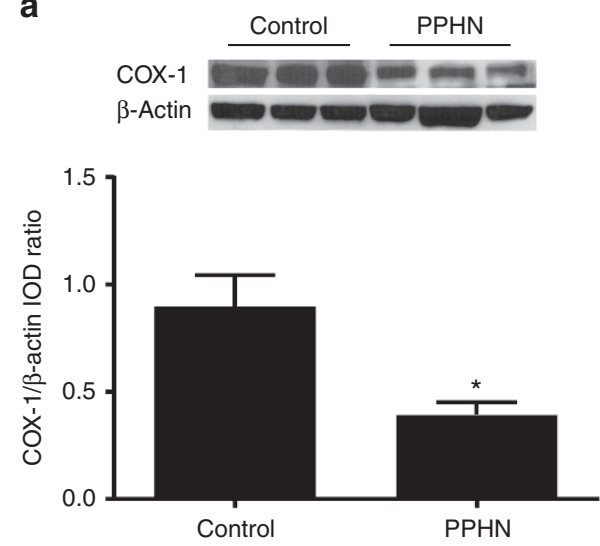

C

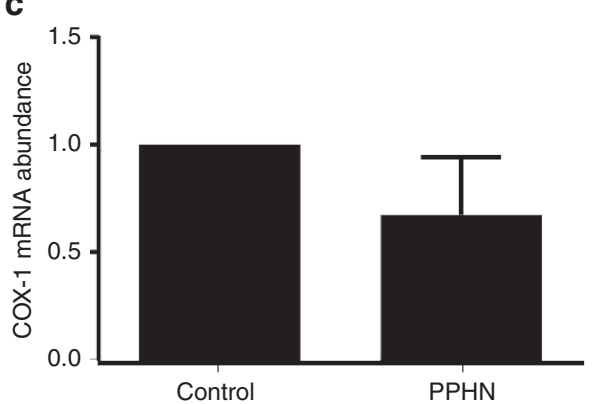

b
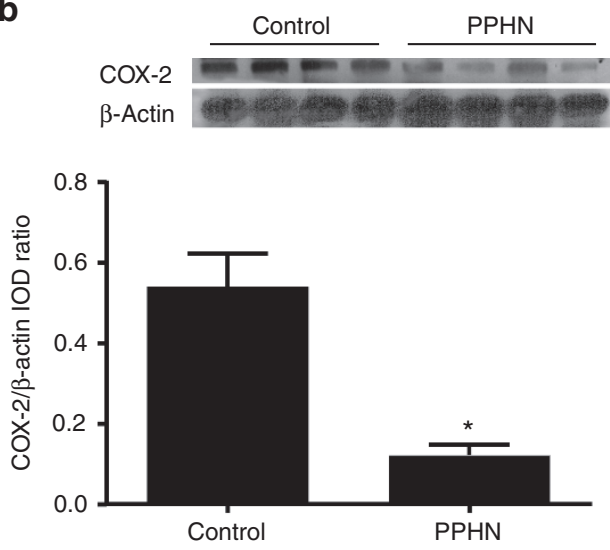

d

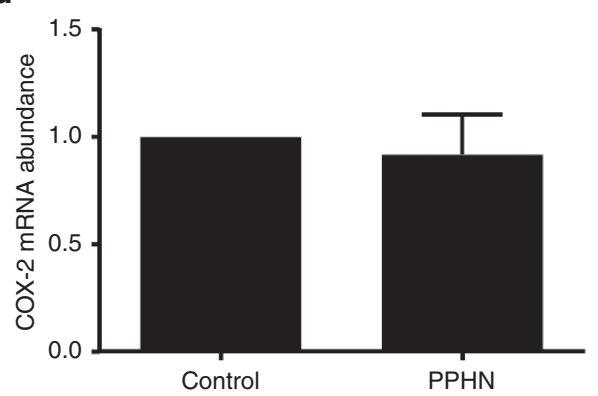

Figure 3. Alterations in protein level of both cyclooxygenase isoforms in PPHN PAEC. (a) Cyclooxygenase-1 (COX-1) and (b) cyclooxygenase-2 (COX-2) protein levels were decreased in PAEC from PPHN lambs $(\mathbf{a}, n=11 ; P<0.05)$. (c) COX-1 and (d) COX-2 mRNA levels were not different between PPHN and control PAEC $(n=8 ; P>0.05) .{ }^{*} P<0.05$ compared with control. PPHN, persistent pulmonary hypertension of the newborn. 
a

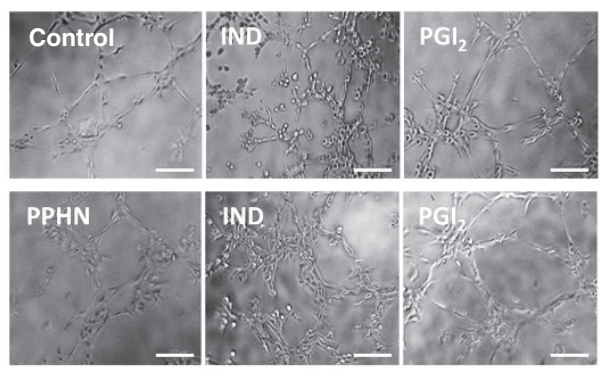

b

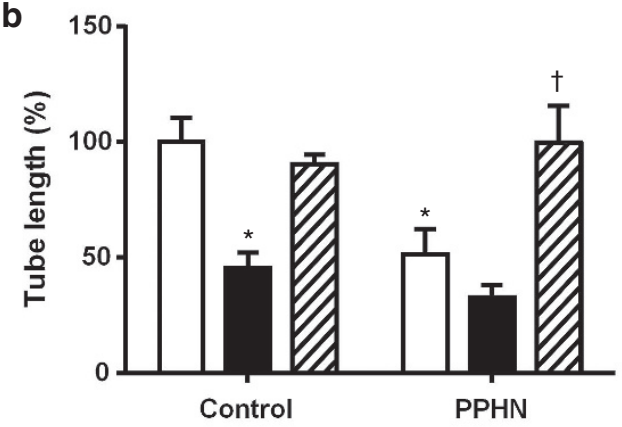

c

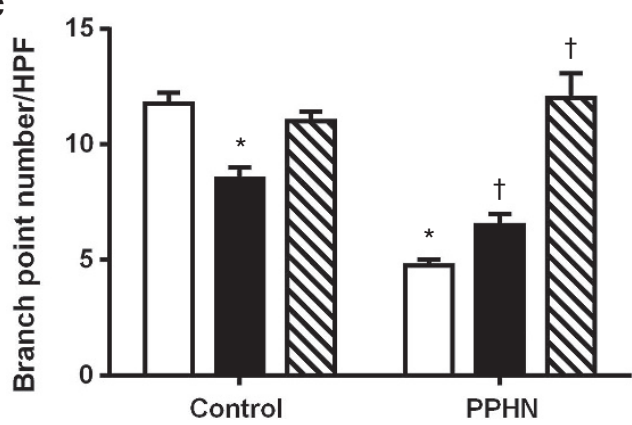

Figure 4. Prostacyclin modulates in vitro angiogenesis. (a) Representative photomicrograph of indomethacin (IND) and $\mathrm{PGI}_{2}$ effects on in vitro tube formation by PAEC from control and PPHN lambs. (b) Tube length and (c) branch point number were decreased in PPHN cells compared with control PAEC. IND decreases tube length ( $\mathbf{a}$ and $\mathbf{b}$ ) and branch point number in control PAEC ( $\mathbf{a}$ and $\mathbf{c}$ ). Decreased tube length ( $\mathbf{a}$ and $\mathbf{b}$ ) and branch point number (a and $\mathbf{c}$ ) in PPHN was improved by adding $\mathrm{PGI}_{2}(100$ $\mathrm{pg} / \mathrm{ml}$ ) to the medium. ${ }^{*} P<0.05$ from untreated control PAEC and from untreated PPHN cells. Open bar, untreated cells; filled bar, indomethacintreated cells, and crosshatch, $\mathrm{PGI}_{2}$-treated cells. Bar $=200 \mu \mathrm{m}$. PPHN, persistent pulmonary hypertension of the newborn.

with $134 \pm 6.4$ in NS-siRNA-treated PAEC under high-power field (Figure $5 c ; n=4 ; P<0.05$ ).

\section{Effect of TXAS Knockdown on Angiogenesis}

Tube length was increased when TXAS was knocked down in control and PPHN cells as compared with NS-siRNA-treated control and PPHN cells (Figure 6a and $\mathbf{b} ; n=10 ; P<0.05$ ). Branch point number was increased in PPHN cells after TXAS knockdown (Figure 6c; $n=10 ; P<0.05$ ). The gap in TXAS knockdown PPHN cells (Figure 6,d,e) and control cells (Figure 6,e) after $6 \mathrm{~h}$ was significantly less on scratch recovery test, indicating that TXAS knockdown has led to faster recovery from injury $(n=20 ; P<0.05)$.

\section{DISCUSSION}

We observed that PPHN is associated with decreased PGIS expression and activity in the PAEC. We also observed a reciprocal increase in the TXAS expression and activity, which suggests a shift of prostanoid metabolism in pulmonary vascular endothelium toward the release of vasoconstrictors over vasodilators in PPHN. Impaired angiogenesis in PPHN may be a downstream effect of decreased PGIS function; exogenous $\mathrm{PGI}_{2}$ restored angiogenesis in PPHN, and knockdown of PGIS in normal cells impaired angiogenesis. Similarly, knockdown of TXAS improved angiogenesis in PPHN.

Several previous studies have mechanistically linked high pulmonary vascular resistance in PPHN to impaired vasodilation and to vascular remodeling $(15,16)$. However, PPHN is also associated with reduced blood vessel density in the lungs (17). Chronic intrauterine pulmonary hypertension impairs lung angiogenesis and alveolar development leading to lung hypoplasia (17). Impairment of angiogenesis can also contribute to increased pulmonary vascular resistance and potentially account for failure of response to vasodilators, such as inhaled NO (6). Therefore, investigation of the mechanisms involved in the impaired angiogenesis in PPHN is critical to improve our current understanding and management of this condition.

Studies of PAEC isolated from fetal lambs with prenatal ductal constriction demonstrated that a decrease in $\mathrm{NO}$ and vascular endothelial growth factor signaling causes impaired in vitro angiogenesis function (1). The role of altered prostaglandin system in the impaired angiogenesis function of PAEC in PPHN, however, remains unclear and motivated our current studies. We observed that the endothelial dysfunction in PPHN includes an alteration in $\mathrm{PGI}_{2}-\mathrm{TXA}_{2}$ signaling in the PAEC and contributes to impaired angiogenesis. $\mathrm{PGI}_{2}$ induces angiogenesis in vivo, via activation of the peroxisome proliferator-activated receptor $\delta$ signaling pathway and upregulation of local vascular endothelial growth factor formation $(7,18,19)$. Our results support a role for $\mathrm{PGI}_{2}$ in the induction of angiogenesis in fetal PAEC. The specific role of $\mathrm{TXA}_{2}$, a vasoconstrictor, in the regulation of angiogenesis function remains unclear with conflicting reports based on the specific vascular bed and condition studied. TXA has been reported to mediate angiogenesis function in the development of tumor metastasis (20). However, TXA receptor activation can also inhibit endothelial cell migration (21). Our study suggests that TXA $\mathrm{T}_{2}$ decreases angiogenesis in fetal PAEC in vitro and may contribute to impaired angiogenesis in PAEC from PPHN lambs.

$\mathrm{PGI}_{2}$ is primarily produced by the vascular endothelium $(22,23)$ and contributes to both vasodilation and angiogenesis (8). PGI $_{2}$ has been identified as a potent pulmonary vasodilator in the fetus and the newborn, and its synthesis is developmentally regulated $(24,25)$. Fike et al. (26) have previously shown that $\mathrm{PGI}_{2}$ levels and PGIS abundance were decreased in piglets with hypoxia-induced pulmonary hypertension, consistent with our observations in the fetal lamb model of PPHN induced by prenatal ductal constriction. We selected this fetal lamb model since previous studies demonstrated that it closely 
a
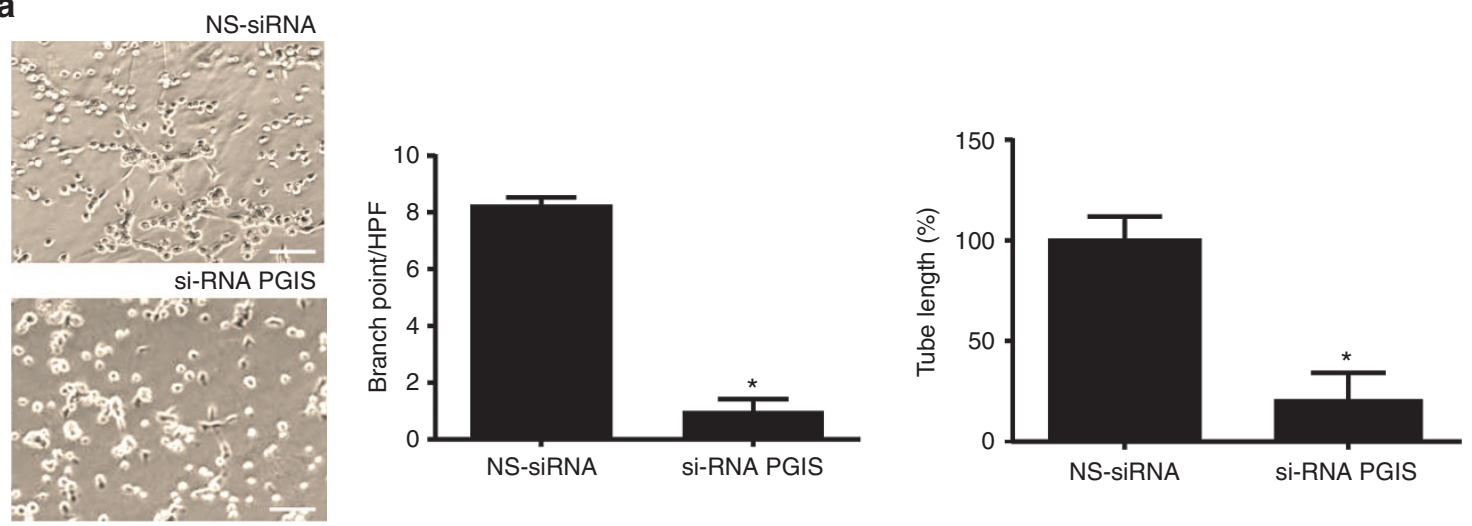

b
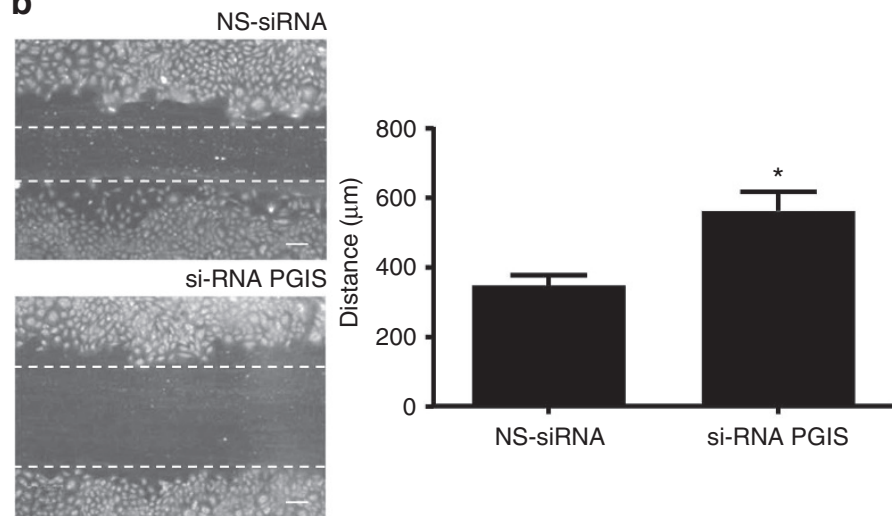

c

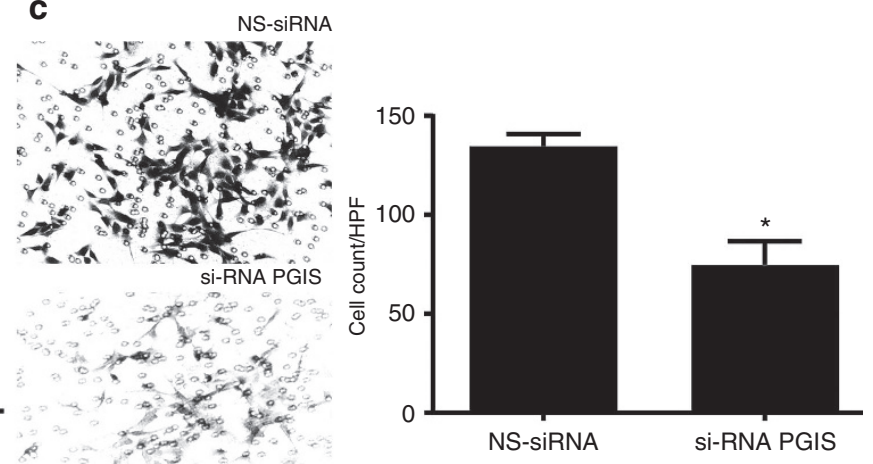

Figure 5. Prostacyclin synthase knockdown impairs angiogenesis in control PAEC. (a) PGIS knockdown with siRNA decreases in vitro tube formation by control PAEC. Branch point number $(n=4 ; P<0.05)$ and tube length $(n=6 ; P<0.05)$ were decreased in control PAEC after application of PGIS-siRNA, compared with nonsilencing (NS)-siRNA. Bar $=200 \mu \mathrm{m}$. (b) PGIS siRNA increased the gap in monolayer of control PAEC after a scratch, indicating longer recovery from injury compared with NS-RNA $(n=10 ; P<0.05)$. Bar $=100 \mu \mathrm{m}$. (c) PGIS siRNA also decreased the number of control cells able to invade through the Boyden chamber as compared with NS-siRNA-treated control cells on cell invasion assay $(n=4 ; P<0.05)$. For all studies, ${ }^{*}$ indicates $P<0.05$ compared with NS-siRNA-treated PAEC. HPF, high-power field; PAEC, pulmonary artery endothelial cell; PGIS, prostacyclin synthase.

simulates PPHN observed in newborn infants $(15,16)$. Our studies suggest that alterations in prostaglandin system are an important component of the impaired angiogenesis observed in this model. Although the decrease in PGI levels in PPHN PAEC was consistent with the decrease in PGIS protein levels, PGIS mRNA did not decrease, suggesting posttranslational regulation of PGIS protein in PPHN.

PPHN is characterized by the presence of increased oxidative stress in PAEC, which can potentially impair angiogenesis $(1,12,27)$. Reaction between superoxide radical and NO generates peroxynitrite, which can cause tyrosine nitration of proteins $(28,29)$. We investigated tyrosine nitration of PGIS, a posttranslational modification that can impair PGIS function, as a potential explanation for the decreased $\mathrm{PGI}_{2}$ levels. PGIS contains tyrosine residue 430, which, when nitrated, decreases the catalytic reaction of the heme-thiolate center of PGIS. Tyrosine nitration of PGIS has been previously shown to reduce its activity $(13,14)$. Increased tyrosine nitration of PGIS in our PPHN cells may also contribute to a decrease in $\mathrm{PGI}_{2}$ levels, leading to endothelial dysfunction and impaired angiogenesis in PPHN. Previous studies have also shown that PGIS nitration in vitro leads to an increase in the levels of $\mathrm{PGH}_{2}$, an intermediate product in the COX pathway, which can be a vasoconstrictor by itself or be a substrate for TXA (30,31). Thus, decreased utilization of $\mathrm{PGH}_{2}$ by PGIS can lead to increased synthesis of TXA, consistent with our observation in this study. We did not measure the levels of $\mathrm{PGH}_{2}$ since it is an unstable transient compound.

Levels of $\mathrm{TXA}_{2}$, a potent vasoconstrictor prostanoid, were elevated in our PPHN cells. In the pulmonary arteries of newborn pigs, chronic hypoxia did not alter the TXA formation $(26,30,31)$. These data point to differences in the mechanism of pulmonary hypertension in the two models. Although we did not observe differences in the basal TXA levels, the ATPstimulated levels were significantly increased in PPHN PAEC. This alteration may be relevant during birth-related transition, which is associated with a surge in ATP levels in pulmonary circulation (32). Although TXAS protein levels were significantly increased in PPHN cells, we observed a decrease in TXAS mRNA levels in PPHN cells. This may be an adaptive response to increased TXAS protein levels in PPHN and suggest posttranslational modifications of TXAS in PPHN.

Our investigation of upstream enzymes in PG pathway was motivated by previous studies showing increased risk of the babies for PPHN following intrauterine exposure to nonsteroidal anti-inflammatory drugs during late gestation (33). One 


\section{Articles | Mahajan et al.}

a

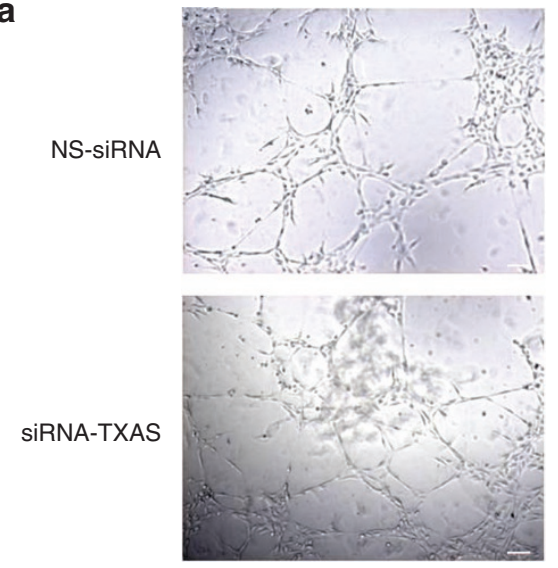

d

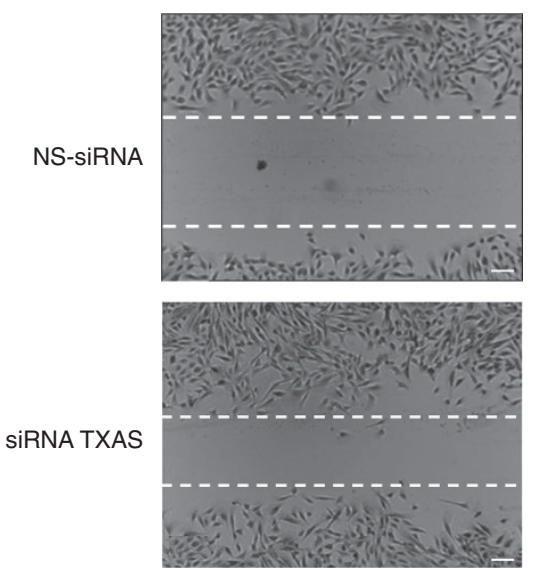

b

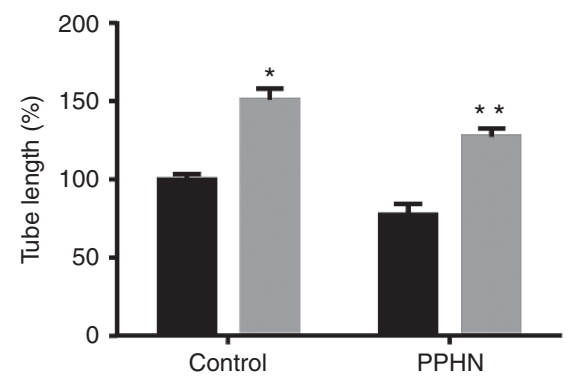

C

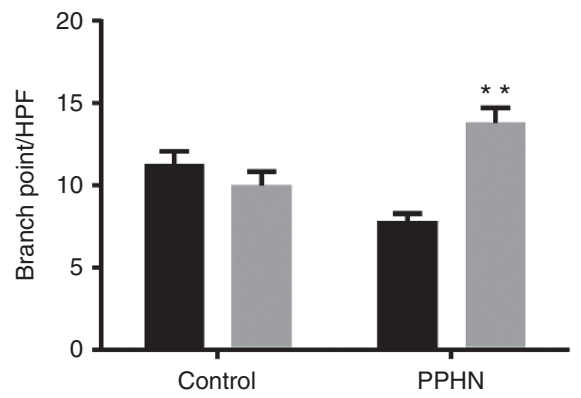

e

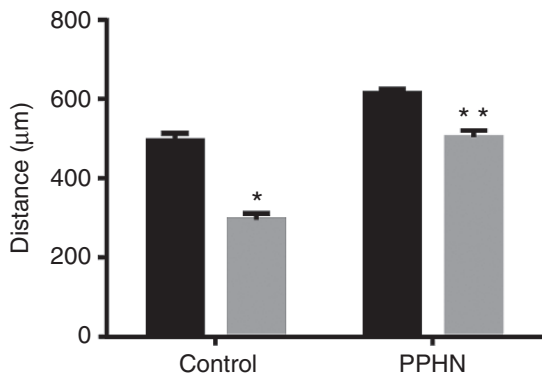

Figure 6. TXAS knockdown improves angiogenesis in PPHN PAEC. (a) TXAS knockdown with siRNA improves tube formation in PPHN cells. Bar $=100$ $\mu \mathrm{m}$. (b) Tube length and (c) branch point number increased significantly after application of TXAS-siRNA, compared with NS-siRNA in both control and PPHN cells $(n=10 ; P<0.05)$. (d) TXAS siRNA also decreased the gap after a scratch in PAEC from PPHN lambs compared with nonsilencing (NS)-siRNA treatment, indicating shorter recovery from injury. Bar $=100 \mu \mathrm{m}$. TXAS knockdown decreased gap distance significantly in both control and PPHN cells $($ e, $n=20 ; P<0.05)$. For all studies, ${ }^{*}$ indicates $P<0.05$ compared with NS-siRNA-treated control cells and ${ }^{* *}$ indicates $P<0.05$ compared with NS-siRNAtreated PPHN cells. Black bar, NS-siRNA and gray bar, TXAS siRNA. PAEC, pulmonary artery endothelial cell; PPHN, persistent pulmonary hypertension of the newborn; TXAS; thromboxane A2 synthase.

of the critical rate-limiting steps in prostanoid production is the conversion of arachidonic acid by COX to prostaglandin $\mathrm{G}_{2}$ and subsequent peroxidation of prostaglandin $\mathrm{G}_{2}$ to $\mathrm{PGH}_{2}$ by the same enzyme. COX-1 is a constitutive enzyme, whereas COX-2 is inducible and may be present only under inflammatory conditions, induction being under transcription control (34). Fike et al. (26) noted increased abundance in COX-1 and no change in COX-2 in hypoxic neonatal pigs. We observed decreased expression of both COX-1 and COX-2 in PPHN cells. The difference in our results may also be due to differences in the animal models used or changes specific to PAEC.

The limitation of our study is that our observations were made in isolated PAEC, and the alterations in PGIS/TXAS signaling may not be directly extrapolated to in vivo lung alterations in infants with PPHN. We also limited our studies to the role of altered PGIS/TXAS signaling in the angiogenesis function of PAEC and did not investigate its role in the vasodilator response of pulmonary arteries.

In conclusion, PPHN is associated with decreased PGIS expression and function and increased TXAS expression and function in PAEC. These reciprocal alterations could potentially explain the decreased angiogenesis previously noted in PPHN. Whether $\mathrm{PGI}_{2}$ restores vasodilatation by improving angiogenesis in neonates with PPHN requires further investigation.

\section{METHODS}

\section{Creation of PPHN Model}

PPHN was induced by prenatal constriction of ductus arteriosus in the fetal lambs from 128 to 136 d gestation, per methods described previously $(16,35)$. Control lambs had thoracotomy and exposure of ductus arteriosus, which was left undisturbed. The study protocol was approved by the Institutional Animal Care and Use Committee of Medical College of Wisconsin. Fetal lungs were harvested at 136 $\mathrm{d}($ term $=140 \mathrm{~d})$ for the isolation of pulmonary arteries and PAEC. Studies were done in PAEC.

\section{Antibodies and Chemicals}

Rabbit anti-PGIS (1:500), anti-COX-2 (1:250), and anti-TXAS antibodies (1:250), mouse anti-COX-1 antibody (1:250), 6-keto prostaglandin $\mathrm{F}_{1 \alpha}$ EIA, and thromboxane $\mathrm{B}_{2}$ EIA Kits were from Cayman Chemical Company (Ann Arbor, MI). PGIS siRNA (bovine), TXAS siRNA (bovine), NS-siRNA, and Immunocruz IP/WB Optima F System were obtained from Santa Cruz Biotechnology (Santa Cruz, CA). Growth factor-reduced Matrigel was from BD Biosciences 
(Bedford, MA). Cell invasion assay kit was obtained from Chemicon International (Temecula, CA). PGIS, TXAS, COX-1, and COX-2 primers were obtained from Invitrogen (Carlsbad, CA). Mouse $\beta$-actin $(1: 1,000)$ and polyclonal nitrotyrosine antibody (Clone 409) and other chemicals were obtained from Sigma Aldrich (St Louis, MO). Polyclonal PGIS antibody for immunoprecipitation was obtained from ProSci (San Diego, CA). Horseradish peroxidaseconjugated anti-mouse or anti-rabbit immunoglobulin $\mathrm{G}$ antibodies $(1: 10,000)$ were obtained from Bio-Rad (Hercules, CA).

\section{Isolation and Culture of Endothelial Cells}

PAEC were isolated with $0.1 \%$ collagenase type A (Roche Molecular Biochemical, Indianapolis, IN) and characterized using techniques described previously (35). Cells for individual experiments were grown to confluence in $100 \mathrm{~mm}$ plates for immunoprecipitation, $60 \mathrm{~mm}$ plates for western blots, 24-well plates for angiogenesis activity assays, 6-well plates for mRNA extraction or transfection, and 24-well plates for $\mathrm{PGI}_{2}$ and TXA 2 assays. We studied PAEC from control and PPHN lambs between passages 4 and 6 .

\section{Western Blot Analysis for Proteins}

PAEC were grown to confluence, and cell lysates were prepared in modified radio-immunoprecipitation assay or 3-(N-morpholino)propansulfonic acid buffer. Protein content was determined by bicinchoninic acid method. A $20-45 \mu \mathrm{g}$ aliquot of protein was resolved by $10 \%$ sodium dodecyl sulfate-polyacrylamide gel electrophoresis. Separated proteins were transferred to nitrocellulose membrane and then blotted with specific antibodies overnight at $4{ }^{\circ} \mathrm{C}$ after blocking with $5 \%$ skim milk in Tris-buffered solution with $0.1 \%$ Tween 20 . Membranes were then blotted with horseradish peroxidase-conjugated anti-mouse or anti-rabbit immunoglobulin $\mathrm{G}$ antibodies. Enhanced chemiluminescence agents were used to visualize the bands. Relative band densities (integrated optic density) were then quantified using Image J (NIH, Bethesda, MD). Readings obtained were normalized to corresponding $\beta$-actin signal, used as the loading control.

\section{Co-immunoprecipitation Studies for PGIS Nitration}

PAEC at $90 \%$ confluence in $100 \mathrm{~mm}$ round dishes were treated with Hank's balanced salt solution for $10 \mathrm{~min}$ (35). The supernatant was then aspirated, and cells were lysed in RIPA buffer. Samples were sonicated, and removal of cell debris was done by centrifugation. PGIS was immunoprecipitated from a $500 \mu \mathrm{g}$ protein aliquot using a specific antibody (35). The immunoprecipitated proteins were separated by sodium dodecyl sulfate-polyacrylamide gel electrophoresis and transferred to nitrocellulose membrane. Membranes were blocked with 5\% skim milk in TBS with $0.1 \%$ Tween 20 and then immunoblotted for PGIS and for nitrotyrosine with specific antibodies. Bands were visualized and quantified as described above. IOD ratio of nitrotyrosine to PGIS was calculated to assess the relative levels of protein nitration.

\section{Measurement of PGIS and TXAS Activity}

Concentrations of 6-keto-PGF1- $\alpha$, the stable metabolite of PGI, and $\mathrm{TXB}_{2}$, the stable metabolite of TXA, were determined in the supernatants of confluent control and PPHN cells by enzyme immunoassay using a commercially available kit (Cayman Chemical). Basal levels of 6-keto-PGF1- $\alpha$ and $\mathrm{TXB}_{2}$ were assessed from supernatant collected over $2 \mathrm{~h}$ from the cells kept in each well of 24 -well plates. 6-Keto-PGF1- $\alpha$ and $\mathrm{TXB}_{2}$ release in response to the physiologic agonist, $\mathrm{ATP}\left(10^{-5}\right)$, was studied after $15 \mathrm{~min}$ exposure, followed by the collection of supernatant. Levels of 6-keto-PGF1- $\alpha$ and TXB were normalized to protein concentration in each well and expressed as picograms/milligram of protein.

\section{Quantification of mRNA Abundance}

PAEC were plated in six-well plates and grown to confluence. RNA extraction was done using RNAeasy mini kit from Qiagen (Germantown, MD). Complementary DNA was synthesized from the extracted RNA using the iScript cDNA synthesis kit (Bio-Rad). The PCR primers were designed using Primer3, and primer sequences are provided online through protocol exchange. Real-time RT-PCR was performed using the iQ5 multicolor real-time PCR detection system (Bio-Rad). The PCR cycle started at $95^{\circ} \mathrm{C}$ for $3 \mathrm{~min}$ followed by 40 cycles of $95{ }^{\circ} \mathrm{C}$ for $15 \mathrm{~s}$ and $58^{\circ} \mathrm{C}$ for $30 \mathrm{~s}$. Melting temperatures were monitored for each pair of primers, and single-peak melting temperature was observed for all of the primer pairs. Number of the threshold cycle $\left(C_{t}\right)$ for each target mRNA was corrected against the corresponding $C_{t}$ of $\beta$-actin mRNA. $2^{-\Delta \Delta C t}$ was then shown as fold change in mRNA abundance with each treatment.

\section{PGIS/TXAS Knockdown}

Knockdown of PGIS and TXAS in control PAEC was accomplished with specific siRNAs (bovine siRNA, sc-270412 and sc-270480; Santa Cruz Biotechnology) and Lipofectamine RNAiMAX Transfection reagent (Invitrogen) using a protocol from the manufacturer. Transfection efficiency was more than $60 \%$ with use of fluorescein isothiocyanateconjugated NS-RNA in previous experiments in our laboratory. After treatment with PGIS siRNA (30 pmoles), TXAS siRNA (30 pmoles), or NS-siRNA and Lipofectamine RNAiMAX $(5 \mu \mathrm{l})$ in six-well plates for $20 \mathrm{~min}$, PAEC $\left(1.5 \times 10^{5}\right)$ were incubated for $48 \mathrm{~h}$ in a humidified $\mathrm{CO}_{2}$ incubator and were then used for performing immunoblots or angiogenesis studies.

\section{Angiogenesis Assays}

In vitro tube formation assay. Tube formation assay was performed in vitro using Matrigel (BD Biosciences, San Jose, CA) in 24-well plates (12). After thawing overnight at $4{ }^{\circ} \mathrm{C}, 300 \mu \mathrm{l}$ of Matrigel was added to designated wells and was incubated for $30 \mathrm{~min}$ at $37^{\circ} \mathrm{C}$ for solidification. PAEC $\left(1.5 \times 10^{5}\right)$ were added on top of the solidified Matrigel. Tube formation by PAEC was monitored over the next $4-6 \mathrm{~h}$ (12). One representative picture was taken per well using an inverted microscope at $\times 20$ (objective) magnification (Nikon Eclipse TE2000; Nikon Instruments, Melville, NY). Total tube length was measured in the presence/absence of $\mathrm{PGI}_{2}(100 \mathrm{pg} / \mathrm{ml})$ at concentrations that simulate physiologic levels. Indomethacin $\left(10^{-5} \mathrm{~mol} / \mathrm{l}\right)$ was then added to some wells, to inhibit PGI release. Data from control and PPHN PAEC were compared. Tube formation was also studied in control PAEC with/without PGIS knockdown and in PPHN cells with/without TXAS knockdown to determine their role in angiogenesis.

Monolayer scratch recovery assay. PAEC with/without PGIS or TXAS knockdown were grown to confluence in six-well plates. Scratch lines were created by a 1-ml pipette tip in each well, and the wells were gently rinsed with HBSS twice to remove the detached cells. The cells were kept in serum-free media for $1 \mathrm{~h}$, followed by addition of Dulbecco's modified eagle medium (DMEM) with $20 \%$ fetal calf serum. The distances between the frontlines of recovery were measured for comparison 4-6h later (12).

Cell invasion assay. Matrigel-coated Transwells with $8-\mu \mathrm{m}$ pores (Chemicon International) were used for the cell invasion assay. Three hundred microliters of serum-free DMEM was placed in each insert and incubated at $37^{\circ} \mathrm{C}$ for $1 \mathrm{~h}$ to soak inserts. The serum-free DMEM was replaced with DMEM containing $1 \times 10^{5} \mathrm{PAEC}$ with/without PGIS knockdown, while DMEM with $20 \%$ fetal calf serum was applied to the outer chamber. The plate was then incubated for $4-6 \mathrm{~h}$. The outer surface of the insert was stained by the staining solution provided in the kit. PAEC that invaded through the 8 - $\mu \mathrm{m}$ pores were counted under the microscope.

\section{Statistical Analyses}

Data are shown as mean \pm SEM. Student's $t$-test was used for normally distributed data, and Mann-Whitney $U$-test was used for data that did not pass the normality test for comparison between two groups. Two-way ANOVA with Student-Newman-Keuls post hoc test was used when more than two groups were compared. Data were analyzed with Graph Pad PRISM software (Graph pad, La Jolla, CA). A P value $<0.05$ was considered statistically significant.

\section{STATEMENT OF FINANCIAL SUPPORT}

Our study was supported by the National Institutes of Health (Bethesda, MD) grants RO3HD-073274 (R.-J.T.), RO3HD-065841 (G.G.K.), and RO1HL-057268 (G.G.K.), as well as CTSI UL1RR031973 from Advancing Healthier Wisconsin Foundation (Milwaukee, WI) grant (R.-J.T. and G.G.K.). G.G.K. was supported by Muma Endowed Chair in Neonatology (Milwaukee, WI).

Disclosure: The authors do not have any financial ties to products in the study or potential/perceived conflicts of interest. 


\section{REFERENCES}

1. Gien J, Seedorf GJ, Balasubramaniam V, Markham N, Abman SH. Intrauterine pulmonary hypertension impairs angiogenesis in vitro: role of vascular endothelial growth factor nitric oxide signaling. Am J Respir Crit Care Med 2007;176:1146-53.

2. Abman SH, Chatfield BA, Hall SL, McMurtry IF. Role of endotheliumderived relaxing factor during transition of pulmonary circulation at birth. Am J Physiol 1990;259(6 Pt 2):H1921-7.

3. Leffler CW, Hessler JR. Perinatal pulmonary prostaglandin production. Am J Physiol 1981;241:H756-9.

4. Velvis H, Moore P, Heymann MA. Prostaglandin inhibition prevents the fall in pulmonary vascular resistance as a result of rhythmic distension of the lungs in fetal lambs. Pediatr Res 1991;30:62-8.

5. Neonatal Inhaled Nitric Oxide Study Group. Inhaled nitric oxide in fullterm and nearly full-term infants with hypoxic respiratory failure. $\mathrm{N}$ Engl J Med 1997;336:597-604.

6. Goldman AP, Tasker RC, Haworth SG, Sigston PE, Macrae DJ. Four patterns of response to inhaled nitric oxide for persistent pulmonary hypertension of the newborn. Pediatrics 1996;98(4 Pt 1):706-13.

7. Gryglewski RJ, Korbut R, Ocetkiewicz A. Generation of prostacyclin by lungs in vivo and its release into the arterial circulation. Nature 1978;273:765-7.

8. Pola R, Gaetani E, Flex A, et al. Comparative analysis of the in vivo angiogenic properties of stable prostacyclin analogs: a possible role for peroxisome proliferator-activated receptors. J Mol Cell Cardiol 2004;36: 363-70.

9. Saito M, Tatsumi K, Kasahara Y, et al. [Effects of thromboxane A2 on chronic hypoxic pulmonary hypertension in the rat]. Nihon Kyobu Shikkan Gakkai Zasshi 1996;34:37-44.

10. Lakshminrusimha S, Porta NF, Farrow KN, et al. Milrinone enhances relaxation to prostacyclin and iloprost in pulmonary arteries isolated from lambs with persistent pulmonary hypertension of the newborn. Pediatr Crit Care Med 2009;10:106-12.

11. Konduri GG, Bakhutashvili I, Eis A, Pritchard K Jr. Oxidant stress from uncoupled nitric oxide synthase impairs vasodilation in fetal lambs with persistent pulmonary hypertension. Am J Physiol Heart Circ Physiol 2007;292:H1812-20.

12. Teng RJ, Eis A, Bakhutashvili I, Arul N, Konduri GG. Increased superoxide production contributes to the impaired angiogenesis of fetal pulmonary arteries with in utero pulmonary hypertension. Am J Physiol Lung Cell Mol Physiol 2009;297:L184-95.

13. Zou MH, Ullrich V. Peroxynitrite formed by simultaneous generation of nitric oxide and superoxide selectively inhibits bovine aortic prostacyclin synthase. FEBS Lett 1996;382:101-4.

14. Zou M, Martin C, Ullrich V. Tyrosine nitration as a mechanism of selective inactivation of prostacyclin synthase by peroxynitrite. Biol Chem 1997;378:707-13.

15. Morin FC 3rd. Ligating the ductus arteriosus before birth causes persistent pulmonary hypertension in the newborn lamb. Pediatr Res 1989;25: 245-50.

16. Abman SH, Shanley PF, Accurso FJ. Failure of postnatal adaptation of the pulmonary circulation after chronic intrauterine pulmonary hypertension in fetal lambs. J Clin Invest 1989;83:1849-58.
17. Grover TR, Parker TA, Balasubramaniam V, Markham NE, Abman SH. Pulmonary hypertension impairs alveolarization and reduces lung growth in the ovine fetus. Am J Physiol Lung Cell Mol Physiol 2005;288:L648-54.

18. Katusic ZS, Santhanam AV, He T. Vascular effects of prostacyclin: does activation of PPAR $\delta$ play a role? Trends Pharmacol Sci 2012;33:559-64.

19. He T, Lu T, d’Uscio LV, Lam CF, Lee HC, Katusic ZS. Angiogenic function of prostacyclin biosynthesis in human endothelial progenitor cells. Circ Res 2008;103:80-8.

20. Nie D, Lamberti M, Zacharek A, et al. Thromboxane A(2) regulation of endothelial cell migration, angiogenesis, and tumor metastasis. Biochem Biophys Res Commun 2000;267:245-51.

21. Ashton AW, Ware JA. Thromboxane A2 receptor signaling inhibits vascular endothelial growth factor-induced endothelial cell differentiation and migration. Circ Res 2004;95:372-9.

22. Shaul PW, Campbell WB, Farrar MA, Magness RR. Oxygen modulates prostacyclin synthesis in ovine fetal pulmonary arteries by an effect on cyclooxygenase. J Clin Invest 1992;90:2147-55.

23. Shaul PW, Pace MC, Chen Z, Brannon TS. Developmental changes in prostacyclin synthesis are conserved in cultured pulmonary endothelium and vascular smooth muscle. Am J Respir Cell Mol Biol 1999;20:113-21.

24. Cassin S, Winikor I, Tod M, et al. Effects of prostacyclin on the fetal pulmonary circulation. Pediatr Pharmacol (New York) 1981;1:197-207.

25. Brannon TS, North AJ, Wells LB, Shaul PW. Prostacyclin synthesis in ovine pulmonary artery is developmentally regulated by changes in cyclooxygenase-1 gene expression. J Clin Invest 1994;93:2230-5.

26. Fike CD, Kaplowitz MR, Pfister SL. Arachidonic acid metabolites and an early stage of pulmonary hypertension in chronically hypoxic newborn pigs. Am J Physiol Lung Cell Mol Physiol 2003;284:L316-23.

27. Wedgwood S, Steinhorn RH, Bunderson M, et al. Increased hydrogen peroxide downregulates soluble guanylate cyclase in the lungs of lambs with persistent pulmonary hypertension of the newborn. Am J Physiol Lung Cell Mol Physiol 2005;289:L660-6.

28. Zou MH. Peroxynitrite and protein tyrosine nitration of prostacyclin synthase. Prostaglandins Other Lipid Mediat 2007;82:119-27.

29. Hink U, Oelze M, Kolb P, et al. Role for peroxynitrite in the inhibition of prostacyclin synthase in nitrate tolerance. J Am Coll Cardiol 2003;42:1826-34.

30. Fike CD, Kaplowitz MR, Zhang Y, Pfister SL. Cyclooxygenase-2 and an early stage of chronic hypoxia-induced pulmonary hypertension in newborn pigs. J Appl Physiol (1985) 2005;98:1111-1118; discussion 1091.

31. Fike CD, Pfister SL, Slaughter JC, et al. Protein complex formation with heat shock protein 90 in chronic hypoxia-induced pulmonary hypertension in newborn piglets. Am J Physiol Heart Circ Physiol 2010;299:H1190-204.

32. Konduri GG, Mattei J. Role of oxidative phosphorylation and ATP release in mediating birth-related pulmonary vasodilation in fetal lambs. Am J Physiol Heart Circ Physiol 2002;283:H1600-8.

33. Alano MA, Ngougmna E, Ostrea EM Jr, Konduri GG. Analysis of nonsteroidal antiinflammatory drugs in meconium and its relation to persistent pulmonary hypertension of the newborn. Pediatrics 2001;107:519-23.

34. Smith WL, Garavito RM, DeWitt DL. Prostaglandin endoperoxide H synthases (cyclooxygenases)-1 and -2. J Biol Chem 1996;271:33157-60.

35. Konduri GG, Ou J, Shi Y, Pritchard KA Jr. Decreased association of HSP90 impairs endothelial nitric oxide synthase in fetal lambs with persistent pulmonary hypertension. Am J Physiol Heart Circ Physiol 2003;285:H204-11. 